\title{
Enhanced recovery after surgery in children
}

\author{
Talha Rafeeqi ${ }^{1}$, Erik G. Pearson ${ }^{2}$ \\ ${ }^{1}$ Department of Surgery, Valley Health System, Las Vegas, NV, USA; ${ }^{2}$ Department of Pediatric Surgery, Sunrise Children's, Las Vegas, NV, USA \\ Contributions: (I) Conception and design: EG Pearson; (II) Administrative support: EG Pearson; (III) Provision of study materials or patients: None; \\ (IV) Collection and assembly of data: All authors; (V) Data analysis and interpretation: None; (VI) Manuscript writing: All authors; (VII) Final \\ approval: All authors. \\ Correspondence to: Erik Pearson, MD, FACS. 653 N. Town Center Blvd Suite \#412, Las Vegas, NV, USA. Email: erikgpearson@gmail.com.
}

\begin{abstract}
Enhanced recovery after surgery (ERAS) is a systematic approach to optimize a patient's health and improve clinical outcomes, increase patient satisfaction and decrease healthcare costs. Enhanced recovery protocols have been used across a variety of surgical disciplines and patient groups to improve patient safety and reduce hospital length of stay without increasing return visits to the system. ERAS involves the application of clinical decision making throughout the patient experience with interventions in the preoperative, perioperative and post operative phases. In addition, ERAS is multidisciplinary and the success of an ERAS program is dependent on the effort and integration of stakeholders across the healthcare system. Utilization of ERAS systems have grown across the global adult surgical community over the last three decades and adoption in pediatric surgery has only occurred recently. Hospitals in both adult and pediatric surgery have found that implementation of ERAS systems lead to a shortened length of stay and reduced complications without increasing patient returns to the system. Importantly patients who have surgery within an ERAS program experience less pain, less opioid utilization, a quicker recovery and increased satisfaction. In pediatric surgery ERAS has successfully been employed across most all disciplines from congenital cardiac surgery to colorectal surgery. The evolution of ERAS continues as a paradigm of quality and safety.
\end{abstract}

Keywords: Enhanced recovery after surgery (ERAS); enhanced recovery protocol; fast track surgery; multimodal pain control

Received: 15 April 2020; Accepted: 24 June 2020; Published: 25 July 2021.

doi: $10.21037 /$ tgh-20-188

View this article at: http://dx.doi.org/10.21037/tgh-20-188

\section{Enhanced recovery after surgery: an evolution of quality and safety}

Enhanced recovery after surgery (ERAS) is a systematic approach to optimize a patient's health and improve clinical outcomes, increase patient satisfaction and decrease healthcare costs. Enhanced recovery protocols have been used across a variety of surgical disciplines and patient groups to improve patient safety and reduce hospital length of stay without increasing return visits to the system (1). Surgery did not always embrace a culture of quality and safety; early in its evolution our profession more closely resembled barbarism. Barber-surgeons practicing in the Middle Ages were dismissed by European medical societies until advances in the late 1800s and early 1900s led to a transformation (2). Specifically, the evolution of surgery as a distinct specialty along with the development of sterile technique, antibiotics, local and regional anesthesia allowed for less painful and increasingly safer procedures. Surgeon-scientists and evidence based practice created a culture of safety and quality and made a concerted effort to challenge dogmatic clinical paradigms. After the Institute of Medicine's report "To Err is Human”, a culture of high quality, safe and patient-centered care evolved quickly (3). This evolution of surgical safety and quality is particularly evident today with the development of ERAS, a patient centered system designed to challenge dogma and replace heuristics with evidence-based care. While ERAS has been with us for over three decades, only recently have we initiated implementation in the pediatric surgical setting. 
ERAS, in its infancy, was implemented as a "fast track" concept by Professor Henrik Kehlet, a gastrointestinal surgeon at Copenhagen University. Dr. Kehlet achieved a median postoperative hospital stay of 2 days for open colectomy patients that were treated in his "fast-track" surgery program (4). This was a significant improvement compared with the standard hospital stay of 5 to 10 days at that time (4). Traditional perioperative and postoperative care promoted prolonged fasting before and after surgical procedures, bowel preparation, extended bed rest, insertion of drainage tubes and catheters, fluid resuscitation and opioid-based analgesia. In Dr. Kehlet's view, these clinical decisions contributed to postoperative deterioration and augmented the stress and inflammatory response precipitated by the surgical insult (4).

Early studies focused on the benefits of regional anesthesia in blunting the surgical stress response demonstrated that optimal perioperative pain relief was associated with improved surgical outcomes $(1,4)$. This seed evolved into a systematic approach reaching beyond pain relief and combined multiple clinical decisions including preoperative coaching, goal setting, health optimization, avoidance of fasting, preoperative carbohydrate loading, multimodal anesthesia, zero fluid balance and postoperative interventions focusing on early mobilization, early enteral intake and opioid-sparing control of postoperative pain. In addition to the techniques employed, the patients eligible for an ERAS program have extended beyond adult colorectal surgery and now include all patients, of all ages, in all settings.

While ERAS is well established across adult surgical disciplines it has only been applied more recently in pediatric surgery. Enhanced recovery pathways in the pediatric population have demonstrated significant benefits in clinical outcomes as well as improved patient and family satisfaction and decreased healthcare costs $(1,5)$. ERAS applied to pediatric surgery includes clinical decision making in the preoperative, perioperative and postoperative settings, is dependent on multiple stakeholders, and extends across all pediatric surgical specialties.

\section{How does an ERAS program function in pediatrics?}

ERAS protocols in pediatrics provide a structured, evidence-based, and multidisciplinary approach to all aspects of perioperative care to optimize a child's recovery from surgery. A child and her family are introduced to
ERAS during the first clinic visit with her surgeon. After an initial assessment the child and parent are given a description of the surgical procedure and ERAS educational material is reviewed with the family including the complete recovery plan. They are educated about goals, expectations, common points of failure, and the multimodal pain plan. Several references are provided to the family including videos and internet resources for their review and all questions are answered. If needed, for example in children with inflammatory bowel disease, the child's health and nutritional status are optimized prior to surgery. On the day prior to surgery, the family receives a phone call with instructions to avoid solid foods after midnight, continue clear liquids until two hours prior to surgery, begin the multimodal pain plan with acetaminophen the night before surgery, and to again answer questions and review goals.

On the day of surgery the child consumes a preoperative carbohydrate-rich drink two hours prior to surgery at home or in the preoperative holding area and is given acetaminophen and gabapentin or celecoxib to attenuate pain and the surgical inflammatory response. During surgery the patient is maintained at zero fluid balance, preemptive anti-nausea medications are given, a regional anesthetic blockade may be administered and minimally invasive surgical techniques are employed. In recovery the child drinks clear liquids and a postoperative multimodal pain regimen continues with opioid-sparing medications including oral acetaminophen, ibuprofen or ketorolac, and gabapentin. The child ambulates on the evening of surgery and goals are reviewed with the family by the nursing staff. The next morning the diet is advanced, a scheduled plan for ambulation is implemented, intravenous fluids are weaned per protocol and safe discharge criteria are reviewed with the family. Prior to discharge follow-up appointments are scheduled and the family is given a goal-oriented activity and recovery plan as well as contact information should they have any questions after leaving the hospital.

Institutional ERAS protocols are developed with contributions from multiple members of the healthcare team and vary by institution according to local demands. Inclusion criteria for ERAS programs differ between hospitals but may include children of all ages undergoing both elective and urgent operations. Enhanced recovery protocols (ERP) have the overall goal of reducing the negative effects of surgical intervention so that patients can recover quickly with fewer complications and have greater satisfaction. The protocols are divided into preoperative, perioperative and postoperative phases. Each of these phases 
has several different components and themes. While each component contributes individually to the success of an ERAS program; the collective integration of all components creates the greatest advantage for patient outcomes $(1,5)$.

\section{The themes and phases of enhanced recovery}

\section{Preoperative phase}

The theme of the preoperative phase is "education and optimization." At the initial clinic visit, the surgeon meets with the patient and family to introduce the ERAS program. This includes explaining to the child and her family that they will take an active role in recovery including early nutrition and ambulation. The multimodal pain plan is explained and the expectations of opioid-free pain control are established with the child and their family. The family is given a handout that emphasizes the information discussed during the clinic visit and asked to watch a short video that takes the child through the entire ERAS experience. The evening before surgery, children are encouraged to undergo a chlorhexidine cleanse and ingest a dose of acetaminophen according to weight-based dosing guidelines to begin the multimodal pain plan. For preoperative fasting, children are encouraged to continue drinking clear liquids up until 2 hours prior to elective surgery while discontinuing any solid food after midnight. Children are encouraged to bring sugar free gum with them to the hospital so that they can begin chewing shortly after surgery as this has been shown to improve gastrointestinal recovery (6).

The second component of the preoperative phase is patient optimization. In adult ERAS programs, preoperative optimization begins with pre-admission risk stratification and patient selection. This may include a scoring system such as the POSSUM (physiological and operative severity scoring for the enumeration of mortality and morbidity) or cardiac risk assessment such as the Lee index $(7,8)$. In children this may or may not be applicable. While most children undergoing elective surgery are healthy, it is important to consider any pre-existing health conditions or behaviors that may adversely affect the surgical outcome. For example, alcohol use and smoking should cease and conditions such as asthma, inflammatory bowel disease, diabetes and nutritional status should be optimized.

While the American Society of Anesthesiologists fasting guidelines currently recommend clear liquids up to two hours before an elective procedure many children are traditionally asked to fast from midnight (9). This leads to increased insulin resistance, patient discomfort and stress, and potentially decreased intravascular volume (10). The practice of fasting from midnight is antiquated and the following guidelines should be used as part of the preoperative phase of an ERAS program: clear liquids should be allowed up to 2 hours, breast milk may be ingested up to 4 hours, and formula, solids and non-human milk up to 6 hours prior to general anesthesia for an elective procedure. In addition to limiting preoperative fasting, ingestion of a carbohydrate drink 2-3 hours prior to the elective procedure has been shown to reduce postoperative insulin resistance, maintain glycogen reserves, decrease protein breakdown and improve muscular strength (11).

The preoperative phase concludes with a checklist audit of all of the preoperative components, a further explanation of the multimodal pain plan preoperatively and answering any questions prior to surgery.

\section{Perioperative phase}

The perioperative phase, which also encompasses intraoperative interventions, emphasizes the theme of "prevention". Specifically, interventions are initiated to prevent postoperative nausea and vomiting (PONV), pain and fluid overload. These goals can be achieved with PONV mitigating strategies, minimally invasive surgery, regional anesthetic blockage, standard anesthesia protocols and avoidance of drainage tubes as appropriate.

The multimodal approach to prevention of PONV in an ERP begins with the reduction of preoperative fasting, adequate hydration and preoperative carbohydrate loading seen in the preoperative phase. In addition, opioid-sparing techniques of the multimodal pain plan including preoperative acetaminophen, non-steroidal antiinflammatory drugs (NSAIDs) and regional anesthesia contribute to decrease the prevalence of PONV. A multimodal approach to pain, especially when regional anesthesia is included, results in significant improvement in time to feeding and opioid utilization (12). Options for regional blockade for abdominal surgery include abdominal trunk blocks such as a transverse abdominal plane (TAP) block, rectus sheath block as well as epidural and spinal analgesia. In some studies these have been shown to be equivalent to epidural blockade, if not superior in terms of lower postoperative nausea/vomiting, ileus and paresthesia (13). With induction and maintenance of anesthesia the anesthesiologist can choose to limit the use of inhalation agents, especially nitrous oxide, and also prophylactically 
treat nausea with dexamethasone and ondansetron.

Standardized anesthetic protocols for intravenous fluids help to prevent fluid overload by giving fluids on the pump at $2-5 \mathrm{~mL} / \mathrm{kg} / \mathrm{h}$, less for laparoscopic surgery and more for open operation (12). Intraoperative fluid therapy should aim to maintain a neutral fluid balance and colloids may be used to treat patients with objective evidence of hypovolemia. A neutral fluid balance helps prevent complications such as pulmonary edema and postoperative adynamic ileus. Maintenance of normothermia is important as several studies have shown that prevention of hypothermia during surgery reduces surgical site infections, cardiac complications, bleeding and transfusion requirements, while improving recovery and overall survival. Intraoperative hypothermia can be avoided by monitoring temperature with nasopharyngeal temperature probes and utilizing active rewarming devices which should be employed in all cases lasting longer than 60 minutes (14). Sequential compression devices should be in place and functioning prior to induction, pre-incision antibiotics should be planned if indicated, and stress-dose steroids may be needed in children who are immunosuppressed.

The choice of surgical technique is important as laparoscopic techniques are well established to decrease length of stay and postoperative morbidity. Minimally invasive techniques also lead to earlier passage of flatus and less narcotic requirements $(15,16)$. Choosing a minimally invasive surgery technique conforms to the philosophy of enhanced surgical recovery by minimizing surgical trauma, wounds and bleeding so that the patient can have a quicker recovery.

\section{Postoperative phase}

The postoperative phase begins in the post anesthesia recovery unit (PACU) and the theme is "rapid recovery". In the PACU, the multimodal pain regimen continues with avoidance of opioid drugs as well as scheduled acetaminophen, ibuprofen or ketorolac, and gabapentin. In addition, a clear liquid diet and gum chewing are started in the recovery unit. Following surgery the patient is encouraged to place cool packs on the wound to limit pain and edema. Ambulation is encouraged at least once on the day of surgery.

One of the major goals of ERAS is to limit postoperative ileus (POI) and advance the patient to tolerance of regular enteral nutrition. There are several ways that ERAS achieves this intraoperatively and postoperatively including the use of minimally invasive surgical techniques, utilization of thoracic epidural analgesia or abdominal trunk blocks, and avoiding excess fluid. Postoperative strategies that assist with limiting POI and advancing diet include regional anesthesia, opioid-sparing strategies with medications such ketorolac and gabapentin, and/or the use of opioid antagonists such as alvimopan or methylnaltrexone. It is clear that multimodal strategies should be utilized for prevention of POI and these strategies are emblematic of an ERAS program.

Early mobilization is a key component of the enhanced surgical recovery pathway and patients that mobilize as part of an ERAS pathway mobilize more and achieve independent mobilization earlier than those patients not on an ERP (17). While there is little data to advise how to achieve early intervention, several strategies such as pedometers, patient diaries, hallway posters, and the absence of in-room entertainment have shown success. Importantly, early mobilization is a goal requiring a multidisciplinary approach with pain control, nursing encouragement and physical therapy.

On post operative day one, ERPs need to ask and answer a few important questions. Can the diet be advanced? Are there any drains, nasogastric tubes, or foley catheters that can be removed? Can intravenous fluids be stopped? Is the patient ready for discharge?

In order for the patient to be discharged safely a discharge checklist should be employed that allows the patient the opportunity to assure themselves and their family that they are ready for a safe discharge. An example follows:

(I) I feel safe going home and I understand my recovery plan.

(II) My pain is controlled on oral medications.

(III) I have all of my prescriptions and understand how to take them.

(IV) I am tolerating clear liquids.

(V) I am able to walk independently or as before surgery.

(VI) I am comfortable with wound care and/or stoma care if applicable.

(VII) My outpatient follow up appointment is arranged and I have the clinic phone number.

(VIII) All of my questions have been answered.

The preoperative, perioperative and postoperative phases all demonstrate essential components of an ERAS program. One of the greatest aspects of an ERAS program that is shared in all three phases is patient engagement (5). Patients should understand from the first clinic visit that 
the success of an ERAS program is not dependent on any particular element or component, rather it is contingent on their engagement with the entire program. Patients and families who read the educational material, take prescribed preoperative medications, optimize their health, hydrate up to 2 hours prior to surgery, ambulate early, advance their diet and engage with the medical team will most likely have a more rapid recovery compared to their unengaged peers.

\section{The Stakeholders in an ERAS program}

The incredible opportunity for hospitals and providers to adopt an ERAS program for their surgical patients will be dependent on a large number of different stakeholders at all levels of hospital operations. The implementation of an ERAS program and its ongoing success extends from surgeons to hospital administration, healthcare providers, nurses, information technologists, educators, stoma therapists, child life specialists, pain psychologists and ancillary staff.

\section{The surgical champion}

The surgery team is fundamental to the implementation and successful operation of an ERAS program. The surgeon champion is the surgeon who leads this charge and is able to understand how an ERAS program will fit within their hospital system. They will work with the information technology department to design the order sets needed for the electronic medical record and provide education to the nurses and staff on the goals of an ERAS program and why it provides substantial benefits to the patient and institution. In addition, the surgeon champion acts as the leader to integrate the other stakeholders toward the common goal of implementing ERAS across all of the pediatric surgical subspecialties.

At each phase of the ERAS program the surgeon is a vital stakeholder. In the preoperative phase the surgeon will be responsible for identifying the patients who would benefit from the ERAS program, educate the patient and their family about ERAS, describe the expectations, plan the surgery and implement the preoperative orders and medications. In the perioperative phase the surgeon works with the anesthesiologist to achieve the operative goals including a minimally invasive approach, zero fluid balance, multimodal pain control, and avoidance of urinary catheters and drainage tubes when possible. Postoperatively the surgeon is responsible for working with nursing staff to ensure that the patient is completing their activity goals, advancing diet, weaning intravenous fluids, and is safe for discharge home.

Successful ERAS programs depend on the surgical team and its commitment to each of the phases' goals as well as the auditing of the ERAS program.

\section{The anesthesia champion}

The anesthesiologist, with the surgeon, is fundamental to the success of an enhanced recovery program. In the preoperative phase the anesthesiologist reviews the plan with the patient and initiates the multimodal pain regimen which includes preoperative medications such as acetaminophen, celecoxib and/or gabapentin as well as nausea and vomiting evaluation and preemptive treatment. In this phase, collaboration between anesthesia and surgery is essential regarding preoperative fasting and the preoperative carbohydrate drink consumption. During the perioperative phase the anesthesiologist continues the multimodal pain program, minimizes narcotic exposure, provides regional analgesic blockade when appropriate and aims for zero fluid balance. In the postoperative phase the anesthesiologist continues the pain plan, minimizes narcotic exposure, prevents nausea, and facilitates recovery in the post-anesthesia recovery unit.

Importantly, the anesthesiologist works with the surgeon to develop the enhanced recovery protocol for the hospital. It is vital that the anesthesiologist be a leader in their group and can communicate with other anesthesiologists so that the protocol is universally adopted.

\section{Nursing teammates}

The nursing team and nursing educators represent the "implementation team" of an enhanced surgical recovery program in any hospital. In the preoperative area the nursing team will be responsible for checking in the patient, going over the care plan and helping to set expectations, administering preoperative medications that were ordered, and giving the preoperative carbohydrate drink if indicated. Postoperatively the nursing team will implement the ERAS order set, assist with activity on the day of surgery, encourage diet advancement and encourage goal setting. This includes confirming that the patient is comfortable and their pain is managed well, that their diet is advancing and intravenous fluids have been weaned or stopped when appropriate. In addition, the nursing team evaluates when 
the patient is safe for discharge by going through the discharge checklist daily.

Communication between the surgery team and the nursing team is critical in the development of an ERAS program. The nursing team should be given education about the goals of enhanced recovery and treated as key teammates in the design and implementation of ERAS.

\section{Administration}

While the surgeon, anesthesiologist and nursing are critical to the development and success of an enhanced recovery program on the clinical side, leadership and "buy-in" at the administrative level is critical for its success within the system. Administrators can help facilitate the development of enhanced surgical recovery protocols in the computerized physician order entry systems (CPOE) of the hospital and help establish the integration of enhanced surgical recovery into the care policies of a hospital. In addition, administrators help facilitate education of the nursing staff and can be a champion for educational and marketing material for the ERAS program. Finally, an administrative champion can help with the audit of an ERAS program through development of an integrated dashboard by working with the information technology team.

\section{The patient as part of the ERAS team}

The patient is an important part of the enhanced surgical recovery team and their commitment to the expectations and goals of the program is required for success. The patient helps to optimize their own health prior to the operation as encouraged and instructed by the surgical team by following the instructions for preoperative medications, preoperative fasting and consuming the preoperative carbohydrate drink when indicated. In addition, the patient and their family are instrumental in the postoperative period by clearly communicating their pain to the nursing and surgical teams, ambulating early and often, chewing gum, advancing diet and taking an active role in their recovery. As the patient returns to their baseline it is critical that they communicate any barriers to recovery to the surgical team and should ask questions early and often if there is any confusion (5,17-19).

\section{The ERAS auditing team}

Implementation of an ERAS program is a significant hurdle to overcome in hospitals given the wide reaching changes asked of the healthcare system. After implementation of ERAS, frequent auditing of the program including a review of past and future patients and discussions with each of the key stakeholders help the system celebrate successes and overcome hurdles $(19,20)$. These hurdles may include specific patient issues such as weaning intravenous fluids, physician education on how to engage with ERAS, and/or implementation training with nurses and therapists. While it is easy to overestimate the adoption of an ERAS program, frequent auditing allows the ERAS team to understand real time data.

\section{Outcomes in pediatric ERAS programs}

Enhanced recovery programs have made significant advancements since the early "fast-track" programs for adult colorectal surgery created and promoted by Dr. Kehlet. ERAS in the adult population and ERPs have transformed the delivery of surgical care by reducing length of stay, hospital readmission and costs $(1,12,17,21)$. This positive transformation is also evident across pediatric surgical specialties with similar successes including reduced length of stay, improved pain control, quicker return of diet and reduced costs.

In 2016, the American Pediatric Surgery Association surveyed pediatric surgeons' practices pertaining to enhanced recovery elements (22). A majority of surgeons surveyed were willing to incorporate $(78 \%)$ or already incorporating (19\%) many ERAS elements in their surgical practice. In addition in 2016, an expert panel of pediatric surgeons, pediatric anesthesiologists, pediatric gastroenterologists, patient representatives and nurse practitioners discussed the adult ERAS pathways and defined the elements that were most critical in the care of pediatric surgical patients (22). Elements of the ERAS protocol at Sunrise Children's Hospital for the preoperative, perioperative and postoperative phases are highlighted in Table 1. The expert panel recommended 19 key elements to be included in an ERAS pathway as appropriate for children. Implementation of these elements have demonstrated reduced length of stay, shortened time to oral intake and reduced opioid utilization $(1,12,17,21)$.

Across pediatric surgery specialties, ERAS and implementation of ERPs have shown tremendous benefits. Following laparoscopic cholecystectomy in children, implementation of an ERP led to a significant increase in same day discharges and reductions in opioid utilization while not increasing the rate of emergency department 
Table 1 Elements of a pediatric ERAS protocol. The most critical ERAS elements in the care of pediatric surgical patients as determined by a survey of an expert panel of pediatric providers and patient representatives are highlighted with an asterix (22)

Pediatric enhanced recovery after surgery protocol

Preoperative Elements

Preoperative ERAS education*

Preoperative multimodal pain plan

Coaching and Expectation Building

Optimize medical comorbidities ${ }^{\star}$

Avoid prolonged preoperative fasting

Administer non-opioid analgesia*

Perioperative Elements

Venous thromboembolism prophylaxis*

Pre-incision antibiotic prophylaxis*

Standard anesthetic protocol ${ }^{\star}$

Regional anesthesia and short acting anesthetic

Minimally invasive technique*

Prevention of nausea and vomiting*

No nasogastric tubes*

Standardized hypothermia prevention*

Postoperative Elements

No intraperitoneal perianastomotic drains*

Goal directed/near-zero fluid therapy*

Early removal of urinary catheters ${ }^{*}$

Prevention of postoperative ileus *

Opioid-sparing pain regimen*

Perioperative nutritional screening*

Early mobilization*

Audit protocol compliance and outcomes *

visits (23). In a population of 51 pediatric patients with inflammatory bowel disease, following implementation of an ERP a significant reduction was observed in morphine utilization and time to oral intake without any increase in postoperative complications (12). In a comparison of a pediatric ERAS cohort with a pre-implementation cohort undergoing elective colorectal surgery, significant decreases were identified in hospital length of stay, time to a regular diet, narcotic utilization and volume of perioperative fluid administered (24).

Benefits of ERPs are not limited to abdominal surgery. Improvement in length of stay, pain tolerance and decreased incidence of urinary catheter usage is demonstrated in patients undergoing corrective surgery for pectus excavatum (25). Out of 109 patients, 58 children in the ERP group experienced a significant decrease in the average length of hospitalization, requirement for urinary catheter placement and pain score. Implementation of an ERP in pediatric and adolescent gynecology demonstrated safety and effectiveness in limiting narcotics and hastening hospital discharge (26). Initial experience with a methadone-based ERP in congenital cardiac surgery demonstrated reduced time on mechanical ventilation and time in the intensive care unit (27).

\section{Conclusion}

ERAS has evolved over the last three decades in adults and children to improve the safety and quality of surgical care delivered across specialties. The pediatric surgery community has embraced ERAS and the implementation of ERPs has been shown to improve children' surgical outcomes, decrease length of stay, and limit opioid use while avoiding increases in complications and return visits to the healthcare system. An ERAS program begins prior to the first patient visit by building robust infrastructure and auditing mechanisms dependent on the input of many important stakeholders. Patient and family engagement and education is important to achieving the key goals of any ERAS program and that is the highest level of safety, quality, value and satisfaction.

\section{Acknowledgments}

Funding: None.

\section{Footnote}

Provenance and Peer Review: This article was commissioned by the Guest Editors (Eduardo Perez, Samir Pandya, and Matthew S. Clifton) for the series "Current Topics in Pediatric General Surgery" published in Translational Gastroenterology and Hepatology. The article has undergone external peer review.

Conflicts of Interest: Both authors have completed the ICMJE uniform disclosure form (available at http://dx. doi. 
org/10. 21037/tgh-20-188). The series "Current Topics in Pediatric General Surgery" was commissioned by the editorial office without any funding or sponsorship. The authors have no other conflicts of interest to declare.

Etbical Statement: The authors are accountable for all aspects of the work in ensuring that questions related to the accuracy or integrity of any part of the work are appropriately investigated and resolved.

Open Access Statement: This is an Open Access article distributed in accordance with the Creative Commons Attribution-NonCommercial-NoDerivs 4.0 International License (CC BY-NC-ND 4.0), which permits the noncommercial replication and distribution of the article with the strict proviso that no changes or edits are made and the original work is properly cited (including links to both the formal publication through the relevant DOI and the license). See: https://creativecommons.org/licenses/by-nc-nd/4.0/.

\section{References}

1. Ljungqvist O, Scott M, Fearon KC. Enhanced Recovery After Surgery: A Review. JAMA Surg 2017;152:292-8.

2. Townsend CM, Beauchamp RD, Evers BM, et al. Sabiston textbook of surgery : the biological basis of modern surgical practice. 20th edition. ed. Philadelphia, PA: Elsevier/Saunders; 2017. p. 2-18.

3. Kohn LT, Corrigan JM, Donaldson MS. To Err is Human: Building a Safer Health System. 2000.

4. Patil S, Cornett E, Jesunathadas J, et al. Implementing enhanced recovery pathways to improve surgical outcomes. J Anaesthesiol Clin Pharmacol 2019;35:S24-8.

5. Heiss KF, Raval MV. Patient engagement to enhance recovery for children undergoing surgery. Semin Pediatr Surg 2018;27:86-91.

6. Meyer JP, Fawcett D. The use of chewing gum for preventing postoperative ileus. BJU Int 2008;101:1-2.

7. Copeland GP, Jones D, Walters M. POSSUM: a scoring system for surgical audit. Br J Surg 1991;78:355-60.

8. Lee TH, Goldman L. Letter by Lee and Goldman regarding article, "Development and validation of a risk calculator for prediction of cardiac risk after surgery". Circulation 2012;125:e385; author reply e386.

9. Practice guidelines for preoperative fasting and the use of pharmacologic agents to reduce the risk of pulmonary aspiration: application to healthy patients undergoing elective procedures: an updated report by the American
Society of Anesthesiologists Committee on Standards and Practice Parameters. Anesthesiology 2011;114:495-511.

10. Holte K, Nielsen KG, Madsen JL, et al. Physiologic effects of bowel preparation. Dis Colon Rectum 2004;47:1397402.

11. Ljungqvist $\mathrm{O}$. Modulating postoperative insulin resistance by preoperative carbohydrate loading. Best Pract Res Clin Anaesthesiol 2009;23:401-9.

12. Phillips MR, Adamson WT, McLean SE, et al. Implementation of a pediatric enhanced recovery pathway decreases opioid utilization and shortens time to full feeding. J Pediatr Surg 2020;55:101-5.

13. Pirrera B, Alagna V, Lucchi A, et al. Transversus abdominis plane (TAP) block versus thoracic epidural analgesia (TEA) in laparoscopic colon surgery in the ERAS program. Surg Endosc 2018;32:376-82.

14. Scott AV, Stonemetz JL, Wasey JO, et al. Compliance with Surgical Care Improvement Project for Body Temperature Management (SCIP Inf-10) Is Associated with Improved Clinical Outcomes. Anesthesiology 2015;123:116-25.

15. Ping L, Yan H, Yang $Y$, et al. Retrospective review of laparoscopic versus open surgery in the treatment of appendiceal abscess in pediatric patients: Laparoscopic versus open surgery for appendiceal abscess. Medicine 2017;96:e7514.

16. Zhang CD, Yamashita $\mathrm{H}$, Zhang S, et al. Reevaluation of laparoscopic versus open distal gastrectomy for early gastric cancer in Asia: A meta-analysis of randomized controlled trials. Int J Surg 2018;56:31-43.

17. West MA, Horwood JF, Staves S, et al. Potential benefits of fast-track concepts in paediatric colorectal surgery. J Pediatr Surg 2013;48:1924-30.

18. Poland F, Spalding N, Gregory S et al. Developing patient education to enhance recovery after colorectal surgery through action research: a qualitative study. BMJ Open 2017;7:e13498.

19. Leeds IL, Boss EF, George JA, et al. Preparing enhanced recovery after surgery for implementation in pediatric populations. J Pediatr Surg 2016;51:2126-9.

20. Harmer M, Davies KA. The effect of education, assessment and a standardised prescription on postoperative pain management. The value of clinical audit in the establishment of acute pain services. Anaesthesia 1998;53:424-30.

21. Yeung SE, Hilkewich L, Gillis C, et al. Protein intakes are associated with reduced length of stay: a comparison between Enhanced Recovery After Surgery (ERAS) and conventional care after elective colorectal surgery. Am J 
Clin Nutr 2017;106:44-51.

22. Short HL, Taylor N, Piper K, et al. Appropriateness of a pediatric-specific enhanced recovery protocol using a modified Delphi process and multidisciplinary expert panel. J Pediatr Surg 2018;53:592-8.

23. Yeh A, Butler G, Strotmeyer S, et al. ERAS protocol for pediatric laparoscopic cholecystectomy promotes safe and early discharge. J Pediatr Surg 2020;55:96-100.

24. Short HL, Heiss KF, Burch K, et al. Implementation of an enhanced recovery protocol in pediatric colorectal surgery. J Pediatr Surg 2018;53:688-92.

doi: $10.21037 /$ tgh-20-188

Cite this article as: Rafeeqi T, Pearson EG. Enhanced recovery after surgery in children. Transl Gastroenterol Hepatol 2021;6:46.
25. Wharton K, Chun Y, Hunsberger J, et al. Successful use of an enhanced recovery after surgery (ERAS) pathway to improve outcomes following the Nuss procedure for pectus excavatum. J Pediatr Surg 2020;55:1065-71.

26. Smith AE, Heiss K, Childress KJ. Enhanced Recovery after Surgery in Pediatric and Adolescent Gynecology: A Pilot Study. J Pediatr Adolesc Gynecol 2020;33:403-9.

27. Iguidbashian JP, Chang P, Lines J, et al. Enhanced recovery and early extubation after pediatric cardiac surgery using single-dose intravenous methadone. Ann Card Anaesth 2020;23:70-4 